\title{
Una base de datos espacial integrada en un Sistema de Información Geográfica para la gestión del terroir: un nuevo sistema consistente e interactivo
}

\author{
Alberto Lázaro-López ${ }^{1}$, María L. González-SanJosé ${ }^{1}$ y Vicente D. Gómez-Miguel ${ }^{2}$ \\ ${ }^{1}$ Dpto. Biotecnología y Ciencia de los Alimentos, Facultad de Ciencias, Universidad de Burgos. Burgos, España. \\ ${ }^{2}$ Dpto. Producción Agraria (Edafología), ETSIAAB, Universidad Politécnica de Madrid, Madrid. España
}

\begin{abstract}
Methodology of the Integrated Zoning of the Terroir involve the generation and management of a high volume of big size data that are interrelated conceptually and spatially, of which its analysis and storage is necessary. These actions are critical in the investigation process and, therefore, there is a special interest in an effectively and efficiently data management. The databases are widely used because of their capabilities on integrity, consistency, redundancy and independence of data management as a method for optimizing resources, so the main goal is to develop a spatial database that facilitates the access, management and analysis of the data of the ZIT. The result is TEZISdb, an acronym for Terroir Zoning Information Service Database, a multilocal and multiscalar modeling that allows to collect thematic and spatial data of all environmental factors from terroir as well as functions for its analysis.
\end{abstract}

Resumen. La Zonificación Integrada del Terroir (ZIT) y su proceso metodológico implican la generación y el manejo de un elevado volumen de datos de gran peso que se encuentran interrelacionados conceptual y espacialmente y el cuál es preciso almacenar y analizar. Estas acciones son críticas en la investigación y, por ello, existe especial interés en manejar los datos eficaz y eficientemente. Las bases de datos (BBDD) se emplean ampliamente por sus capacidades en integridad, consistencia, redundancia e independencia en la gestión de datos como método para la optimización de recursos, por lo que se plantea como objetivo el desarrollo de una BD espacial que facilite el acceso, manejo y análisis de los datos procedentes de la ZIT. El resultado es TEZISdb, acrónimo de Terroir Zoning Information Service Database, un modelado multilocalización y multiescalar que permite recoger datos temáticos y espaciales de todos los factores ambientales que conforman el terroir así como funciones para su tratamiento y análisis.

\section{Introducción}

La técnica de zonificación se utiliza frecuentemente, y cada vez con mayor precisión, como una herramienta adecuada para el conocimiento, descripción, delimitación y cartografía de los terroir. La zonificación fue definida en 2003 por Fregoni y col. [1] como "la investigación para el estudio del territorio con el fin de repartirlo en zonas relativamente homogéneas como interacción entre el viñedo y el ambiente" y permite trabajar sobre los elementos con influencia en el desarrollo de los viñedos y en la calidad del vino de forma conjunta.

De los distintos sistemas desarrollados para zonificar, actualmente se utilizan métodos que promueven el empleo de modelos complejos [2], que combinan mapas temáticos e información espacial de diferente índole, y que han sido utilizados con éxito en distintas DO españolas [3].

A través de esta metodología se estudia, cuantifica y cartografía un amplio rango de parámetros de los diferentes factores implicados en la definición del medio (clima, litología, geomorfología, suelo, vegetación y usos del suelo) junto con datos asociados al cultivo (variedad, portainjerto, distribución del viñedo, densidad de plantación, producción...). Su análisis permite seleccionar los parámetros y variables que se consideran de mayor influencia en la diferenciación zonal y constituye la Zonificación Ambiental que proporciona el mapa de las "Unidades Homogéneas del Medio" (UHM). Posteriormente, la relación de estas unidades con los productos (uva, mosto, vino) procedentes de ellas da lugar a la Zonificación Integrada del Terroir (ZIT) [4].

Durante todo este proceso se utilizan intensivamente datos procedentes de teledetección, como el Modelo Digital del Terreno (MDT o DEM en inglés) o imágenes multi-espectrales, de los que son derivados nuevos índices en formato de imágenes ráster de alta resolución espacial y espectral, así como datos georreferenciados expresados en geometrías (puntos, líneas o polígonos) con atributos asociados. Esto supone la generación y el manejo de un elevado volumen de datos de gran peso que se encuentran interrelacionados conceptual y espacialmente, de los cuales es preciso su análisis 
atendiendo a dicho aspecto para generar nueva información, así como su almacenamiento, con el objetivo de preservarlos y permitir que estén disponibles para futuras aplicaciones que permitan derivar nuevos usos y obtener nuevas conclusiones. Estas acciones son críticas y, por ello, existe especial interés en manejar los datos eficaz y eficientemente.

De forma generalizada, la manera de abordar esta situación ha sido mediante una gestión basada en un sistema de ficheros con archivos individuales y de diferentes tipos organizados manualmente. Sin embargo, esto lleva asociadas ciertas contrapartidas: la carencia de integridad y consistencia en los datos, al no poder asegurarse que un valor es coherente con el atributo al que representa ni que un mismo valor sea representado regularmente de la misma forma; la existencia de redundancia, al generarse duplicidad cuando se establecen relaciones; la definición de estructuras rígidas, que dificultan su actualización; la falta de independencia entre la estructura de los datos y las consultas que se quiera hacer sobre ellos; y la falta de optimización de estas consultas. Además, la interoperabilidad entre diversos tipos de archivos no siempre está garantizada, supone una capa añadida de complejidad y dificulta la sistematización de los flujos.

Alternativamente, a partir de la búsqueda de superación de estas dificultades surgen las bases de datos (BBDD) y los sistemas gestores de BBDD (SGBD), que aportan ventajas en integridad, consistencia y no redundancia de los datos; una gestión con una organización independiente del sistema de ficheros; y en el análisis, con búsquedas optimizadas y desligadas de la estructura [5]. Estas capacidades han hecho que su uso se haya extendido a multitud de campos y, en concreto, han sido ampliamente utilizadas para gestionar datos pertenecientes a recursos naturales y del medio como el suelo, la vegetación, meteorología y clima, hidrología, sanidad vegetal, etc [6]. Aun así, no ha sido posible reconocer ninguna referencia bibliográfica de BBDD específicas para el terroir.

\section{Antecedentes}

El concepto de BD se refiere a una colección de datos especialmente organizados para lograr búsquedas $\mathrm{o}$ disposiciones con posterioridad [7] y, para cumplir con estos requisitos, se desarrollan modelos de BBDD que pretenden alcanzarlos desde diferentes perspectivas. En unos sistemas prima la eficiencia, creando estructuras orientadas al modo de consulta. En 1970 se introdujo un nuevo sistema, el modelo relacional de BBDD, que centró su atención en la consistencia y la integridad de los datos sobre la eficiencia.

Según este concepto, las entidades físicas reales podían asimilarse a tablas relacionadas entre sí, para sostener, de esa manera, datos relativos a ellas. Tomando como base el modelo de BD relacional se desarrolló posteriormente el modelo objeto-relacional como una evolución que incorporaba conceptos del modelo orientado a objetos, como herencias y tipos de datos complejos y definidos por el usuario, aportando aún mayor flexibilidad en la construcción de estructuras y robustez [8].

Ejemplo de datos complejos son los datos espaciales, que reúnen en un único atributo información de geometría, sistemas de referencias y sistemas de coordenadas. Estos datos cuentan con un estándar de almacenamiento y acceso recogido en la norma ISO 19125, denominada Simple Feature Access (SFA) [9].

La definición de los datos espaciales como atributo abrió la posibilidad de diseñar bases de datos espaciales que reúnen en un mismo espacio de trabajo datos temáticos y georreferenciados y permiten su tratamiento conjunto. Esto supuso un nuevo paradigma que superaba a los modelos previos de separación de $\mathrm{BD}$, para datos exclusivamente temáticos, y herramientas SIG, encargadas del tratamiento de los georreferenciados, que habían predominado desde el origen de las herramientas SIG en la década de 1960. Este hecho se ve reforzado por la mejora de los motores de los SGBD que soportan el modelo objeto-relacional, con la incorporación también de funciones con las que manipular los datos georreferenciados.

Esta transición queda patente, por ejemplo, en el crecimiento de lo que hoy día es el National Soil Information System (NASIS) [10] y los productos que incluye desarrollados por la National Soil Survey Cooperative en los EUA. Tiene sus inicios en unas herramientas SIG junto a una BBDD relacional y, en la actualidad, ha adoptado como herramienta para la gestión de datos una BBDD con un sistema de modelado objetorelacional con soporte de datos georreferenciados [11]. De forma similar, se ha producido el desarrollo de otras BBDD espaciales para la gestión de recursos naturales [6].

Tomando en cuenta las consideraciones previas sobre la zonificación del terroir, que implica un elevado volumen de datos temáticos y georreferenciados combinados, y las ventajas asociadas a la utilización de BBDD, el empleo de una BBDD espacial se intuye como una herramienta crítica para el avance cualitativo en la investigación del terroir. Sin embargo, no se ha identificado ninguna referencia bibliográfica relacionada. 
Una BD espacial integrada en un SIG para la gestión del terroir: un nuevo sistema consistente e interactivo

Por ello, se establece como objetivo general, facilitar la gestión y el acceso a los datos de la ZIT, mediante los objetivos particulares de desarrollar un modelado para todos los datos ligados a la zonificación del terroir, implementarlo en una BBDD y generar funciones de análisis para el terroir. Como método de comprobación y validación, el diseño será aplicado a la zonificación del terroir de la DO Campo de Borja.

\section{Material y métodos}

\subsection{Fuente de los datos}

La DO Campo de Borja comprende 65.296,20 ha localizadas en la provincia de Zaragoza, al noreste de la Península Ibérica y cuenta con 6.242,89 ha de viñedo, de las que $3.442,69$ ha (2015) se dedican a Garnacha. Los datos utilizados proceden del proyecto de zonificación de la DO [4] y se centra en las UHM de la variedad Garnacha caracterizadas y cartografiadas durante el estudio [12].

\subsection{La estructura de los datos}

Para el desarrollo de una BD se ha seguido el estándar de arquitectura ANSI, que contempla tres fases progresivas: Conceptual, Lógica y Física.

Los requisitos generales establecidos durante la fase Conceptual contemplan que la $\mathrm{BD}$ sea interconectable con diversas aplicaciones, posibilitando tanto el acceso como la edición; extensible, permitiendo incorporar nuevos módulos de funcionalidad en el futuro; y de código libre, facilitando que los avances realizados puedan ser compartidos con la comunidad científica. Desde el punto de vista del contenido específico, el modelado para la zonificación del terroir debe aglutinar datos de parámetros provenientes de los diferentes factores ambientales del terroir, a saber, geología y geomorfología, suelo, clima, vegetación, uso del suelo.

De estos factores existen estructuras de datos ya conceptualizadas $y$ en funcionamiento adaptadas a diferentes áreas, lo que haría de una BD para la zonificación del terroir una suerte de agrupación que integrase las BBDD de los factores. Esto ha servido de apoyo para la elaboración del diagrama ER y su posterior transformación a modelo lógico empleando un sistema objeto-relacional.
Se ha tomado como referencia para el suelo, geología y geomorfología la teoría expuesta en el Soil Survey Manual [13] [14] y las estructuras de SOTER [15] y NASIS [10]. Para la vegetación y uso del suelo se ha adoptado la nomenclatura y organización del Mapa Forestal de España y SIGPAC y el Catastro respectivamente [16].

Para la implementación física se ha utilizado PostgreSQL en su versión 10, un SGBD de tipo servidor-cliente, de código libre, que cumple con las propiedades ACID y que se integra con múltiples extensiones. Entre ellas PostGIS, una extensión para datos espaciales tanto vectorial como ráster que cumple con la norma SFA y cuenta con numerosas funciones de manipulación y consulta, empleada en su versión 2.4. Como apoyo para la visualización se ha empleado la aplicación SIG QGIS, también de código libre, con un amplio soporte para PostgreSQL.

Al encontrarse el desarrollo de la BD en fase de prototipo, no se ha establecido perfiles ni grupos de acceso específicos al no estar planteada actualmente su distribución.

\section{Resultados}

El resultado es una BD para la zonificación del terroir que ha sido denominado TEZISdb, acrónimo de Terroir Zoning Information Service Database. Se trata de un modelado multilocalización y multiescalar, extensible a cualquier región de interés y a cualquier escala que aloja datos temáticos y espaciales de todos los factores ambientales que conforman el terroir.

Se ha optado por diferenciar tantos esquemas como factores se han descrito, resultando en los esquemas de geología y geomorfología (geo) (Figura 1), suelo (suelos) (Figura 2), clima (clima), vegetación (vege) y usos (usos); esquemas auxiliares como base cartográfica (base) y un esquema propio para los resultados del estudio (terroir). Cada uno de estos esquemas agrupan tablas principales con soporte en tablas auxiliares e índices y vistas sobre dichas tablas. 


\section{Web of Conferences}

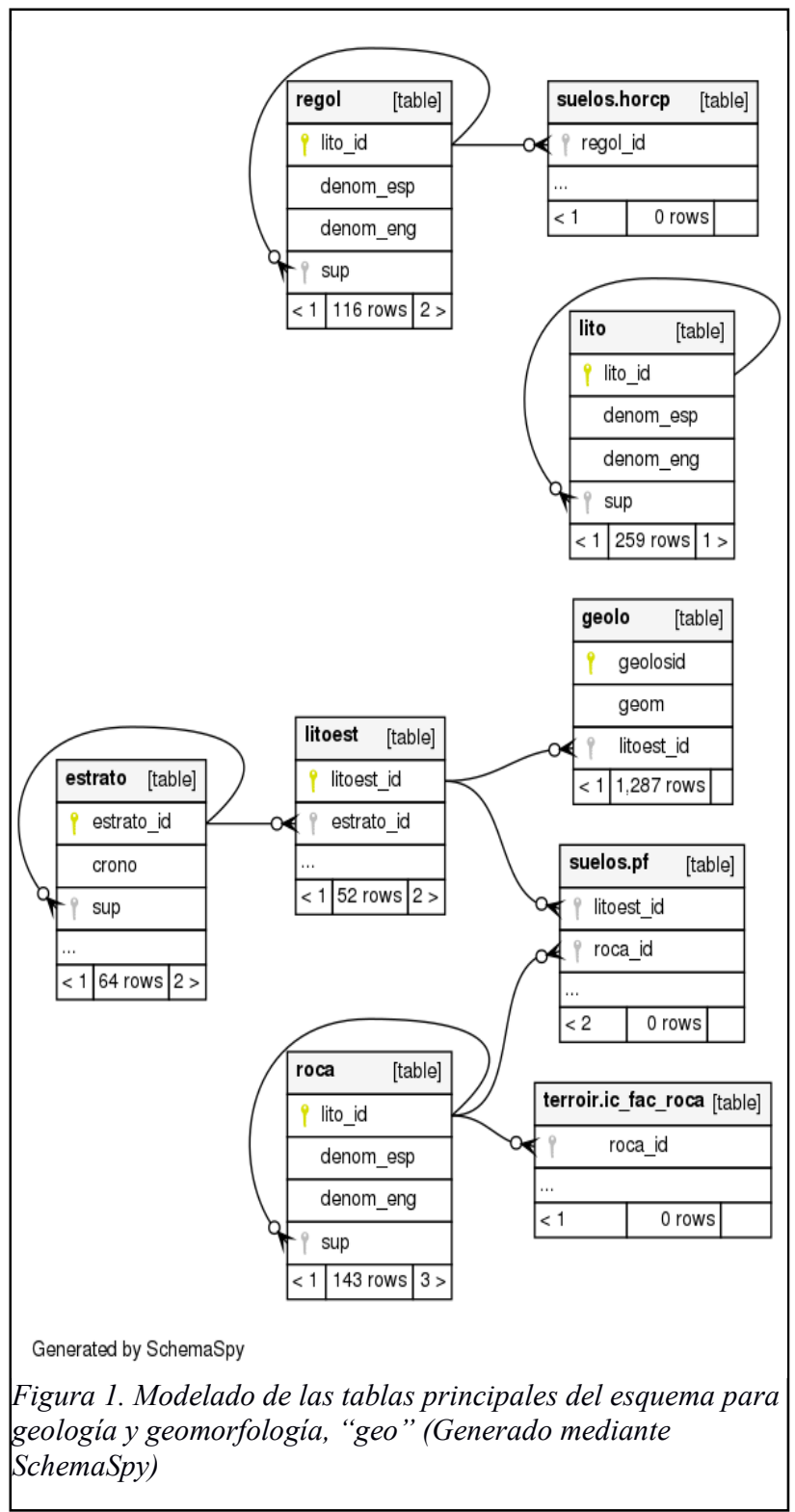

Las principales funciones incluidas están relacionadas con el cálculo de índices, ya sean índices bioclimáticos, edáficos o varios que intervienen en la caracterización de las UHM o el Índice de Calidad del terroir. Dichas funciones de consulta y análisis de datos están desarrolladas principalmente bajo SQL, estando integradas en los esquemas.

La validación del modelado desarrollado a través de su aplicación a la zonificación del terroir de en la DO Campo de Borja ha resultado satisfactoria. Ha conseguido alojar todos los datos que componen el proyecto de zonificación, con una distribución de 11 tablas para el esquema "geo"; 26 en "clima"; 6 para "vege"; 8 en "usos"; 10 en "base", 9 en "terroir" y, por último, 58 tablas para "suelos", además de diferentes vistas y vistas materializadas. Es relevante que el tamaño final de la BD supone menos del 10\% del total del conjunto de archivos que formaban el proyecto de zonificación original, considerando versiones, subversiones y uniones pertenecientes al flujo de trabajo.

También, durante la carga de los datos ha sido posible identificar y corregir ciertos fallos con valores que no cumplían las restricciones establecidas en el diseño, permitiendo verificar la integridad y consistencia de los datos de la zonificación en la DO Campo de Borja al final del proceso. Los fallos encontrados se centraban fundamentalmente en errores de transcripción y nomenclatura.

El uso de esta nueva herramienta y la metodología asociada ha conseguido reducir los tiempos de cálculos y procesos en el flujo de trabajo, lo que establece una nueva marca de eficiencia para la técnica de zonificación del terroir, dejando abierta la posibilidad de nuevas mejoras en el código que los redujeran aún más. 


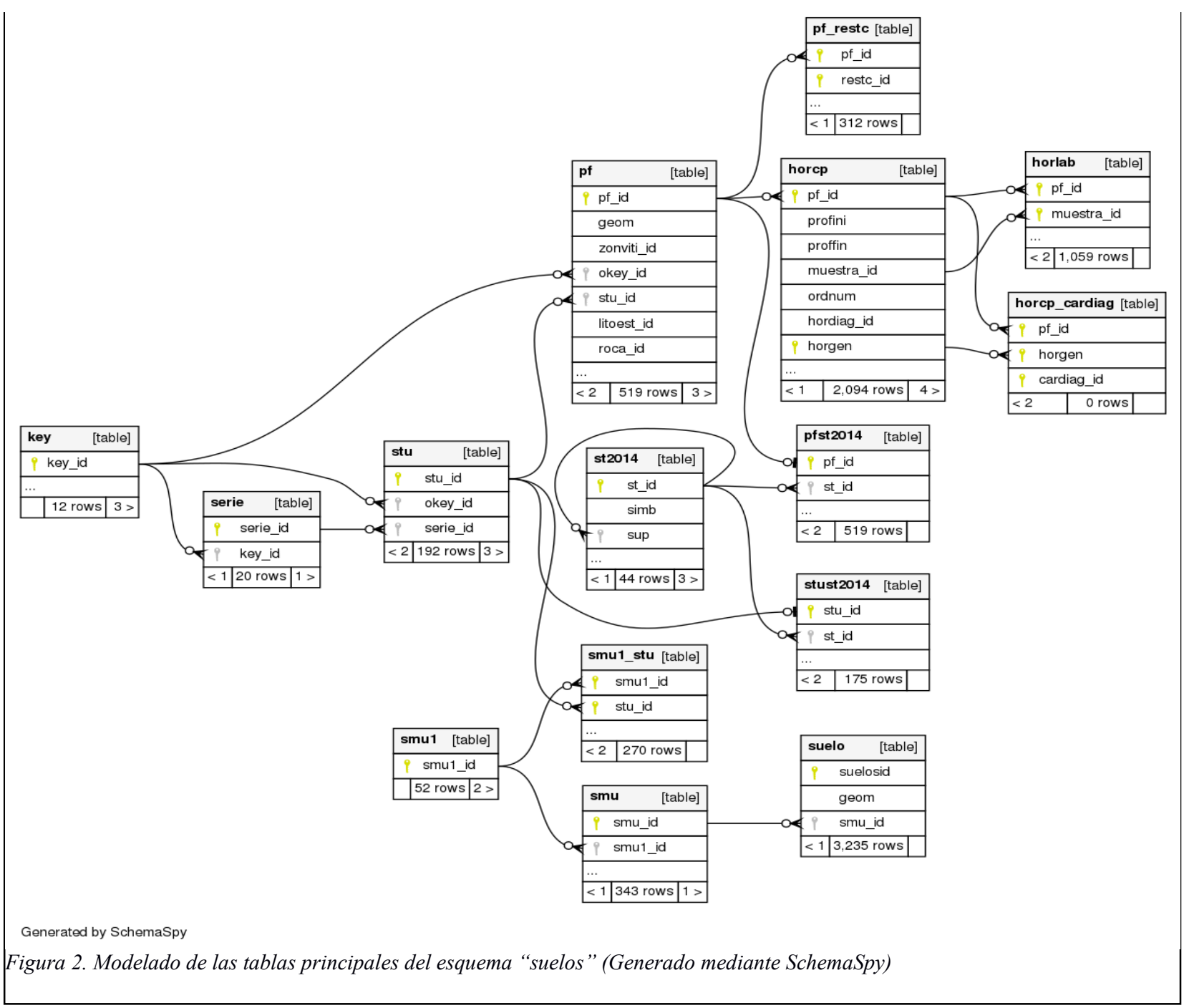

\section{Conclusiones}

Se ha diseñado un modelado de BD espacial para la ZIT denominado TEZISdb, acrónimo de Terroir Zoning Information Service Database, que permite recoger datos temáticos y espaciales de todos los factores ambientales que conforman el terroir, extensible a cualquier región de interés y escala. Ha sido desarrollado bajo un modelo objeto-relacional para el que se ha elegido como SGBD PostgreSQL con su extensión PostGIS, que lo habilita para la gestión y el análisis de datos espaciales.

Esta herramienta permite implantar un nuevo paradigma de flujo de trabajo con implicaciones sobre:

La integridad, consistencia y redundancia de los datos, al almacenar sólo los datos originales mientras se aplican restricciones y comprobaciones automáticas con lo que se evitan múltiples copias dispersas y se optimiza el espacio empleado.

La gestión y análisis de los datos, al centralizar el mantenimiento y control de acceso a los datos sobre el SGBD y desligando las consultas de las estructuras. Esto posibilita la conexión de múltiples aplicaciones que realizan consultas complejas y la definición de funciones de análisis de datos en diversos lenguajes que automatizan cálculos de la ZIT.

El modelo ha sido aplicado a la zonificación de la DO Campo de Borja como método de comprobación y validación, dando como resultado una BD TEZISdb funcional para esa región.

Si bien con la BD se logra una gestión y análisis de los datos más robusto y sistemático, la interacción con ella queda limitada a aquellos técnicos y científicos con conocimientos específicos en manejo de bases de datos y SIG, por lo que resulta interesante disponer de un sistema visual, accesible e intuitivo, que facilitase información actualizada y adaptada para cada perfil de usuario.

Una línea de desarrollo futuro contemplada es el diseño de una Infraestructura de Datos Espaciales (IDE), que sume, a la gestión de los datos con TEZISdb, una interfaz de interacción con usuarios (UI) y diferentes canales de comunicación vía telemática, creando una experiencia de acceso unificada a los datos del terroir. Actualmente se trabaja sobre una UI que se apoya en tecnologías disponibles en visualización de datos SIG, personalizándola para los datos del terroir: como consultas directas sobre las delineaciones del mapa para obtener información de las SMU, la composición de STU, la descripción de cada STU y los perfiles 
característicos definidos para cada STU. También la información conjunta de todos los factores para cada UHM.

\section{Bibliografía}

[1] M. Fregoni, D. Schuster, y A. Paoletti, Terroir, zonazione, viticoltura : trattato internazionale. Verona: Phytoline, 2003.

[2] OIV, «Resolución 423/2012: OIV Guidelines for vitiviniculture zoning methodologies on a soil and climate level». OIV/VITI, 22-jun-2012.

[3] V. D. Gómez-Miguel, «Inventarios del Recurso Suelo de las DO de Castilla y León. Parte I: Importancia, metodología y aplicaciones», Innovación y Tecnología Agroalimentaria, vol. 2, p. 14, nov-2007.

[4] V. D. Gómez-Miguel y V. Sotés, «Zonificación del Terroir: Estudio de Suelos y Ordenación del Cultivo de la Vid en la DO Campo de Borja (Zaragoza)», Universidad Politécnica de Madrid (UPM), Madrid, 2015.

[5] N. Matthew y R. Stones, Beginning Databases with PostgreSQL: From Novice to Professional, Second edition. 2005.

[6] P. M. Huang, Y. Li, y Sumner, Eds., Handbook of Soil Sciences: Resource Management and Environmental Impacts. CRC Press, 2011.

[7] C. J. Date, The new relational database dictionary: a comprehensive glossary of the concepts arising in connection with the relational model of data, with definitions and illustrative examples. Sebastopol, CA: O’Reilly Media, 2015.

[8] R. O. Obe y L. S. Hsu, PostgreSQL: Up and Running, Second edition. Beijing: O'Reilly, 2015.

[9] R. O. Obe y L. S. Hsu, PostGIS in Action, Second edition. Shelter Island, NY: Manning, 2015.

[10] Soil Survey Division Staff, «Chapter 1: Understanding NASIS», en NASIS User Guide, Version 6.1., USDA-Natural Resources Conservation Service, 2011, p. 1:19.

[11] D. E. Beaudette y A. T. O'Geen, «SoilWeb: 13 Years Later», ISRIC Spring School 2016.

[12] A. Lázaro-López, J. Cámara, Á. Martínez, V. D. Gómez-Miguel, y V. Sotés Ruiz, «Terroir Zoning in Appellation Campo de Borja (Northeast Spain): Homogeneous Terroir Units (HTU) and Terroir», presentado en International Terroir Congress, 11th, McMinnville, OR (USA), 2016, p. 452:457.

[13] Soil Survey Division Staff, Soil Survey Manual. Washington, D.C.: U.S. Government Printing Office, 2017.

[14] A. Van Wambeke y T. Forbes, Guidelines for using Soil Taxonomy in the names of soil map units. Soil Conservation Service. US Department of Agriculture (USDA), 1986.

[15] V. W. P. van Engelen y J. A. Dijkshoorn, Global and National Soils and Terrain Digital Databases (SOTER), 2.0. ISRIC. World Soil Infomation, 2013. [16] Instituto Geográfico Nacional, «Geoportal IDEE». [En línea]. Disponible en: http://idee.es/. [Accedido: 18abr-2018]. 